\title{
Low Concentration of Fecal Valeric Acid at 1 Year of Age Is Linked with Eczema and Food Allergy at 13 Years of Age: Findings from a Swedish Birth Cohort
}

\author{
Monica Gio-Batta ${ }^{a}$ Karin Spetz ${ }^{b}$ Malin Barman ${ }^{c, d}$ Lennart Bråbäck ${ }^{e}$ \\ Elisabeth Norin ${ }^{f}$ Bengt Björkstén ${ }^{g}$ Agnes E. Wold ${ }^{a}$ Anna Sandin ${ }^{b}$ \\ anstitute of Biomedicine, Department of Infectious Diseases, Sahlgrenska Academy, University of Gothenburg, \\ Gothenburg, Sweden; 'b Department of Clinical Science, Pediatrics, Sunderby Research Unit, Umeå University, Umeå, \\ Sweden; 'Department of Biology and Biological Engineering, Food and Nutrition Science, Chalmers University of \\ Technology, Gothenburg, Sweden; 'Institute of Environmental Medicine, Unit of Metals and Health, Karolinska Institutet, \\ Stockholm, Sweden; 'Section of Sustainable Health, Department of Public Health and Clinical Medicine, Umeå University, \\ Umeå, Sweden; fDepartment of Microbiology, Tumor and Cell Biology, Karolinska Institutet, Stockholm, Sweden; 9Institute \\ of Environmental Medicine, Karolinska Institutet, Stockholm, Sweden
}

\section{Keywords}

Allergy · Childhood · Eczema · Food allergy · Short-chain fatty acids

\begin{abstract}
Background: Short-chain fatty acids (SCFAs) are abundant bacterial metabolites in the gut, with immunomodulatory properties. Hence, they may influence allergy development. Previous studies have linked fecal SCFA pattern during infancy with allergy. However, the association of SCFAs to allergic outcomes in adolescence is not well established. Here, we examined how the fecal SCFA pattern at 1 year of age related to allergy at 13 years of age. Methods: Levels of 8 SCFAs in fecal samples collected at 1 year of age from 110 children were quantified using gas chromatography. The same individuals were evaluated at 13 years of age for allergic symptoms, allergy diagnosis and allergy medication by questionnaire, and for sensitization using skin prick test against egg, milk, fish, wheat and soy, cat, dog, horse, birch, and timothy grass. Results: The concentration of fecal valeric acid at 1 year of age was inversely associated with eczema at 13 years of age (OR $0.6,95 \% \mathrm{Cl}: 0.4-1.0, p=0.049)$ and showed
\end{abstract}

a trend for inverse association with food allergy at 13 years of age (OR 0.6, 95\% Cl: 0.4-1.0, $p=0.057$ ). In a sub-group analysis of children with eczema at 1 year of age, a higher concentration of fecal valeric acid was linked with reduced risk of their eczema remaining at 13 years of age (OR 0.2, 95\% $\mathrm{Cl}$ :0.0-1.5), although this latter analysis did not reach statistical significance ( $p=0.12)$. Conclusions: Our findings lend further support to the notion of early childhood as a critical period when allergy may be programmed via the gut microbiota. Higher levels of fecal valeric acid may be characteristic of a protective gut microbiota and/or actively contribute to protection from eczema and food allergy.

(C) 2021 The Author(s)

Published by S. Karger AG, Basel

\section{Introduction}

The prevalence of allergic diseases has increased dramatically over the last century in industrialized countries $[1,2]$. Migration and adoption studies make it clear that the tendency to become allergic is chiefly determined in

Edited by: Irina Lehmann, Berlin. karger@karger.com www.karger.com/iaa

Karger $\frac{1}{\%}$

BOPEN ACCESS
C) 2021 The Author(s)

Published by S. Karger AG, Basel

This is an Open Access article licensed under the Creative Commons Attribution-NonCommercial-4.0 International License (CC BY-NC) (http://www.karger.com/Services/OpenAccessLicense), applicable to the online version of the article only. Usage and distribution for commercial purposes requires written permission.
Correspondence to:

Monica Gio-Batta, monica.gio-batta@gu.se

Anna Sandin, anna.sandin@umu.se 
infancy, even when the actual allergic disease may present at school-age or later $[3,4]$. Early exposure to pathogenic microbes via the oral route is associated with protection from allergic disease [5-7]. However, the commensal microbiota may also play a role in maturation of the immune system and protection from allergy. Infants in Western countries are less rapidly colonized by commensal gut bacteria and have a slow turnover of individual bacterial strains, as compared with infants in low-income countries $[8,9]$. Further, there has been a gradual change in the early gut colonization pattern, with reduced prevalence of typical fecal bacteria such as E. coli and Bacteroides, and increased prevalence of typical skin bacteria, notably Staphylococci, that expand in the infant gut due to decreased competition from more "professional" gut bacteria $[9,10]$.

A microbiota of low complexity in early infancy appears to be associated with increased risk of later development of allergy [11-13]. In addition, the composition of the gut microbiota at the species level during the neonatal period has also been related to allergy development [14]. Furthermore, there appears to be a critical window in early childhood, when the gut microbiota is established in parallel with the early development of the immune system, during which the risk of developing allergic disease is programmed. However, the specific features of the gut microbiota that either promote allergy or protect against it remain unclear, limiting the development of preventative strategies.

Short-chain fatty acids (SCFAs), composed of between 2 and 6 carbon atoms $\left(\mathrm{C}_{2}-\mathrm{C}_{6}\right)$, are abundant bacterial metabolites in the large intestine. They have certain immunoregulatory properties and may act as a link between the gut microbiota and the immune system $[15,16]$. SCFAs are produced when undigested carbohydrates and protein from the diet, as well as intestinal mucous and sloughed epithelial cells, are metabolized by the colonic microbiota [16]. The SCFA pattern changes considerably over the first 2-3 years of life. Initially acetic acid $\left(\mathrm{C}_{2}\right)$ dominates, but SCFAs containing higher numbers of carbon atoms increase with time $[17,18]$, in parallel with the development of an increasingly complex, anaerobic gut microbiota [19]. Propionic and butyric acids $\left(\mathrm{C}_{3}-\mathrm{C}_{4}\right)$ are common in the large intestine, while SCFAs such as valeric acid $\left(\mathrm{C}_{5}\right)$ are found at substantially lower levels [20].

In previous studies based on the BAS (BarnAllergiStudien or Pediatric Allergy Study) birth cohort, we observed that infants who were allergic at 1 year of age had lower concentrations of propionic, iso-butyric, butyric, iso-valeric, and valeric acid in their feces than infants who were not allergic at this age [21]. Conversely, they also had higher concentrations of iso-caproic acid. Low concentrations of fecal iso-butyric, valeric and iso-valeric acid at 1 year of age were also associated with onset of food allergy between 1 and 4 years of age [22]. These findings suggest a delayed or impeded development of the complex, primarily anaerobic gut microbiota that establishes over the first 2-3 years of life in healthy children, as discussed previously $[21,22]$. Further, we have recently shown that farming children have a more "mature" SCFA pattern than control children, with higher concentrations of fecal iso-butyric, valeric, and iso-valeric acid at 3 years of age [18]. Children who grow up on farms have low risk of developing allergy and SCFA may be a part of the allergy-preventive effect of farm living $[18,23]$.

Despite this, epidemiological evidence for the role of SCFAs in allergy remains unclear. Regarding eczema, for instance, one study observed higher levels of propionate and butyrate at 3 months of age in infants with eczema by 18 months, but lower levels of the same SCFAs at 6 months [24]. In contrast, lower concentrations of propionate and butyrate were observed throughout the first year in infants with eczema by 3 years [25], and also in a mixed agegroup of eczemateous patients [26], while lower levels of fecal butyrate and valerate, but not propionate, were observed in 6-month-old eczemateous infants [27]. Further, another study found no difference in fecal SCFA levels between 3-month-old infants who later developed eczema and controls [28]. Evidence with respect to food allergy [29-32], rhinitis and asthma [33, 34], and sensitization $[34,35]$ is similarly mixed. Furthermore, few studies have included follow-up periods up to adolescence. In this study, our objective was to investigate associations between the fecal SCFA pattern at 1 year of age and allergic symptoms at 13 years of age.

\section{Methods}

Study Design

This study is based on a subgroup of 110 children participating in the BAS (BarnAllergiStudien or Pediatric Allergy Study) prospective birth cohort, which targeted all 1,228 infants born at Östersund hospital in northern Sweden between February 1996 and January 1997. Further details from the birth cohort have been described previously $[21,22,36]$.

Children were followed longitudinally, with allergy assessment and skin prick tests against common allergens carried out at 1, 4, and 13 years of age $[21,22]$. At 1 year of age (11-18 months), fecal samples were requested from children with a positive skin prick test at 1 year of age $(N=71)$ and also from a sample of children with a negative test $(N=224)$. Samples were obtained from 139 children, 28 with a positive and 111 with a negative skin prick test, and these 
Table 1. Characteristics of the study population and the cohort from which they derived

\begin{tabular}{lllr}
\hline & $\begin{array}{l}\text { Study population } \\
(N=110), \%(n / N)\end{array}$ & $\begin{array}{l}\text { Cohort participants at 13 years } p \text { value } \\
\text { of age }(N=834), \%(n / N)\end{array}$ \\
\hline Sex (female) & $42(46 / 110)$ & $50(407 / 807)$ & 0.09 \\
Older sibling(s) & $61(67 / 110)$ & $52(484 / 782)$ & 0.84 \\
Exclusive breastfeeding at 4 months & $74(80 / 108)$ & $75(561 / 748)$ & 0.84 \\
Antibiotics by 1 year & $24(26 / 110)$ & $26(203 / 783)$ & 0.61 \\
Maternal allergy & $44(48 / 109)$ & $43(359 / 829)$ & 0.89 \\
Allergy at 13 years & $56(61 / 110)$ & $41(338 / 834)$ & 0.01 \\
Eczema at 13 years & $26(28 / 110)$ & $19(154 / 833)$ & 0.08 \\
Food allergy at 13 years & $26(28 / 110)$ & $18(149 / 834)$ & 0.06 \\
Allergic asthma at 13 years & $17(19 / 110)$ & $10(76 / 786)$ & $<0.01$ \\
Allergic rhino-conjunctivitis at 13 years & $29(32 / 110)$ & $18(143 / 786)$ & 0.05 \\
Respiratory allergy at 13 years & $24(26 / 110)$ & $32(249 / 786)$ & $<0.01$ \\
Sensitization at 13 years & $49(54 / 110)$ & & 0.01 \\
\hline
\end{tabular}

The study population derived from the population-based BAS birth cohort $(N=1,231) ; 68 \%$ were examined at 13 years of age $(N=834)$. SCFAs were analyzed in 139 children in the original cohort at 1 year of age, 110 of whom were evaluated for allergy at 13 years of age, and hence constituted the study population examined here. SCFAs, short-chain fatty acids; BAS, BarnAllergiStudien.

were analyzed for SCFAs. In addition, parents answered a questionnaire regarding gestational age at birth, birth weight, breastfeeding, siblings, infections, and use of antibiotics. At 13 years of age, adolescents who still lived in the region filled in a questionnaire together with their parents $(n=834,68 \%)$ regarding symptoms, treatment and diagnosis of allergies, as well as parental allergies, siblings and pet ownership throughout childhood. Questions related to allergy symptoms were based on the International Study of Asthma and Allergies in Childhood questionnaire (ISAAC) [37]. Skin prick tests were carried out on 794 (65\%) adolescents.

The population analyzed in this study included those children who provided a fecal sample at 1 year of age, and answered the questionnaire and underwent a skin prick test at 13 years of age $(n=110)$. The characteristics of the study population are shown in Table 1.

\section{Allergy Definitions}

Allergy at 13 years of age was defined as eczema, allergic asthma, allergic rhino-conjunctivitis, or food allergy using responses to allergy-related questions in the study questionnaire, in certain cases complemented by the skin prick test response. Eczema was defined as pruritic, chronic, or chronically relapsing noninfectious dermatitis with typical features and localization, experienced in the last 12 months. Food allergy was defined as reported symptoms of food allergy. Allergic asthma was defined as reported doctor's diagnosed asthma and/or use of asthma medication and/or wheezing symptoms during the last 12 months. In addition, sensitization (a positive skin prick test) to airborne allergen(s) was required. Allergic rhino-conjunctivitis was defined as itchy eyes and/or runny nose and sneezing upon contact with an airborne allergen during the last 12 months. Sensitization to airborne allergen(s) was required. Respiratory allergy was defined as allergic asthma, as defined above or runny nose and sneezing upon contact with an airborne allergen during the last 12 months, together with sensitization to relevant allergen(s). Non-allergic was defined as no reported allergic symptoms and no sensitization.
The prevalence of allergic manifestations in the study population $(N=110)$ and in the part of the wider BAS cohort that was examined at 13 years of age $(N=834)$ is shown in Table 1 . In total, 61 adolescents had at least one allergic condition, while 33 were non-allergic, which also included not being sensitized to any common allergen. Seventeen adolescents were excluded as they had symptoms suggestive of allergy, but did not fulfill the criteria. The allergic individuals included 28 cases of eczema, 28 cases of food allergy (19 of whom were diagnosed after 1 year of age), 19 cases of allergic asthma, 32 cases of allergic rhino-conjunctivitis, and 26 cases of respiratory allergy (Table 1).

\section{Skin Prick Test}

Skin prick tests were performed using standardized extracts of wheat, egg, milk, soy, fish, cat, dog, horse, birch, and timothy grass (Solu-Prick; ALK, Hørsholm, Denmark). Histamine dihydrochloride $10 \mathrm{mg} / \mathrm{mL}$ was used as a positive control. Peanut allergen was excluded due to the risk, though small, of severe allergic reaction. Tests were carried out on the volar aspect of the forearm, by the same nurse each time. The participants were instructed to avoid antihistamines for $72 \mathrm{~h}$ before the test. A positive test was defined as a mean wheal diameter of at least $3 \mathrm{~mm}$ after $15 \mathrm{~min}$.

\section{Determination of SCFAs in Feces}

Fecal SCFA levels at 1 year of age were determined previously [21, 22]. In brief, parents collected fecal samples at home and these were frozen at $-20^{\circ} \mathrm{C}$. For analysis, samples were thawed and $0.5 \mathrm{~g}$ of feces was mixed with $2 \mathrm{~mL}$ of distilled water containing $0.5 \mathrm{mmol} / \mathrm{L}$ of sulfuric acid and $3 \mathrm{mmol} / \mathrm{L}$ of 2 -ethylbutyric acid as an internal standard. Eight SCFAs (acetic, propionic, butyric, iso-butyric, valeric, iso-valeric, caproic, and iso-caproic acid) were determined using gas chromatography. The detection level was $0.2 \mathrm{mmol} / \mathrm{kg}$ feces. SCFA levels were expressed as concentration, in millimoles per kilogram, and proportion, defined as the concentration of the individual SCFA as a percentage of the total SCFA concentration. 
Table 2. Prevalence, concentration and proportion of fecal short-chain fatty acids at 1 year of age

\begin{tabular}{lllll}
\hline SCFA & $\begin{array}{l}\text { Molecular } \\
\text { structure }\end{array}$ & $\begin{array}{l}\text { Detected, } \\
N(\%)\end{array}$ & $\begin{array}{l}\text { Concentration (mmol/kg; } \\
\text { median and range) }\end{array}$ & $\begin{array}{l}\text { Proportion (\% of total SCFA } \\
\text { concentration; median and range) }\end{array}$ \\
\hline Acetic & $\mathrm{n}-\mathrm{C}_{2}$ & $110(100)$ & $63(9-176)$ & $61(23-84)$ \\
Propionic & $\mathrm{n}-\mathrm{C}_{3}$ & $110(100)$ & $16(2-49)$ & $16(3-38)$ \\
Butyric & $\mathrm{n}-\mathrm{C}_{4}$ & $110(100)$ & $15(2-65)$ & $15(4-38)$ \\
Iso-butyric & $\mathrm{i}-C_{4}$ & $107(97)$ & $1.4(<0.2-7.1)$ & $1.4(<0.2-11)$ \\
Valeric & $\mathrm{n}-C_{5}$ & $78(71)$ & $0.5(<0.2-5.6)$ & $0.5(<0.2-3.8)$ \\
Iso-valeric & $\mathrm{i}-C_{5}$ & $108(98)$ & $1.6(<0.2-7.7)$ & $1.5(<0.2-18)$ \\
Caproic & $\mathrm{n}-C_{6}$ & $17(16)$ & $<0.2(<0.2-2.8)$ & $<0.2(<0.2-3.0)$ \\
Iso-caproic & $\mathrm{i}-C_{6}$ & $29(26)$ & $<0.2(<0.2-3.8)$ & $<0.2(<0.2-2.4)$ \\
\hline
\end{tabular}

SCFAs in a fecal sample collected at 1 year of age were examined using gas chromatography. Individual SCFAs were expressed as median concentration, as well as percentage of the total SCFA concentration (proportion). $N=$ 110. SCFAs, short-chain fatty acids. ${ }^{\dagger}$ Defined by the number of atoms in the carbon chain $\left(C_{n}\right)$ and whether it is straight ( $\mathrm{n}-$ ) or branched (i-).

\begin{abstract}
Statistical Analyses
$\chi^{2}$ tests were used to compare characteristics between the current study population and the wider BAS cohort. Logistic regression analysis was used to identify associations between host factors or fecal SCFA levels at 1 year of age and allergy at 13 years of age. Analyses relating to specific allergic manifestations included all cases, and therefore individuals with multiple manifestations were represented in $>1$ group. Non-allergic individuals constituted the reference group, apart from in the subgroup analysis containing only individuals with eczema at 1 year of age in which the outcome was eczema/no eczema at 13 years. Linear regression analysis was used to identify associations between host factors and fecal SCFA concentration. Data were analyzed using SPSS statistics software (IBM SPSS statistics data editor, version 25). $p$ values $<0.05$ were considered statistically significant.
\end{abstract}

\section{Results}

Fecal SCFAs at 1 Year of Age and Allergy at 13 Years of Age

Fecal SCFA levels were previously determined for 110 participants in the BAS birth cohort who provided a fecal sample at 1 year of age $[21,22]$. In this study, their SCFA pattern was examined for associations with allergy at 13 years of age. In general, the individuals examined here differed little from the background population of adolescents examined for allergy at 13 years of age, as shown in Table 1, although rates of allergy and sensitization were higher. This was due to the fact that fecal samples were requested from children at 1 year of age based on skin prick test results, in order to accumulate more allergy cases in this group. The fecal SCFA pattern observed at 1 year of age in 139 children in the BAS cohort has been previ-

SCFAs in Infancy and Allergy in

Adolescence ously reported [22], and the results for the 110 individuals included here is shown in Table 2. As seen in the Table, acetic, propionic, and butyric acid comprised $>90 \%$ of the total SCFA concentration and each of them could be detected in all children. Valeric acid and the branched SCFAs iso-butyric and iso-valeric acid together constituted $<5 \%$ of the total SCFA concentration and were detected in most children (Table 2). Caproic and iso-caproic acid were detected in a minority of children and in low levels.

The fecal SCFA concentration at 1 year of age was related to allergy at 13 years of age, as shown in Table 3. Individuals who were non-allergic at 13 years of age constituted the reference group. The concentration of valeric acid at 1 year of age was inversely associated with eczema at 13 years of age (OR 0.6, 95\% CI: $0.4-1.0, p=0.049$ ) and also showed a trend for inverse association with food allergy (OR 0.6, 95\% CI: 0.4-1.0, $p=0.057$ ). The concentration of iso-caproic acid at 1 year of age was consistently and positively linked with all allergic manifestations evaluated in this study at 13 years of age (OR 1.5-2.1), although the associations did not reach statistical significance. As well as the concentration of fecal SCFAs, the proportion of fecal SCFAs in relation to the total SCFA concentration was also determined. The proportion of fecal valeric acid at 1 year of age was inversely associated with eczema and food allergy at 13 years of age (OR 0.6, 95\% CI: $0.4-1.0, p=0.046$ and OR 0.5, 95\% CI: $0.3-0.9$, $p=0.03$, respectively).

Nine adolescents who were food allergic at 13 years of age were already diagnosed with food allergy at 1 year of age. This could have prompted the parents to alter their diet, leading to a changed fecal SCFA pattern, as the 


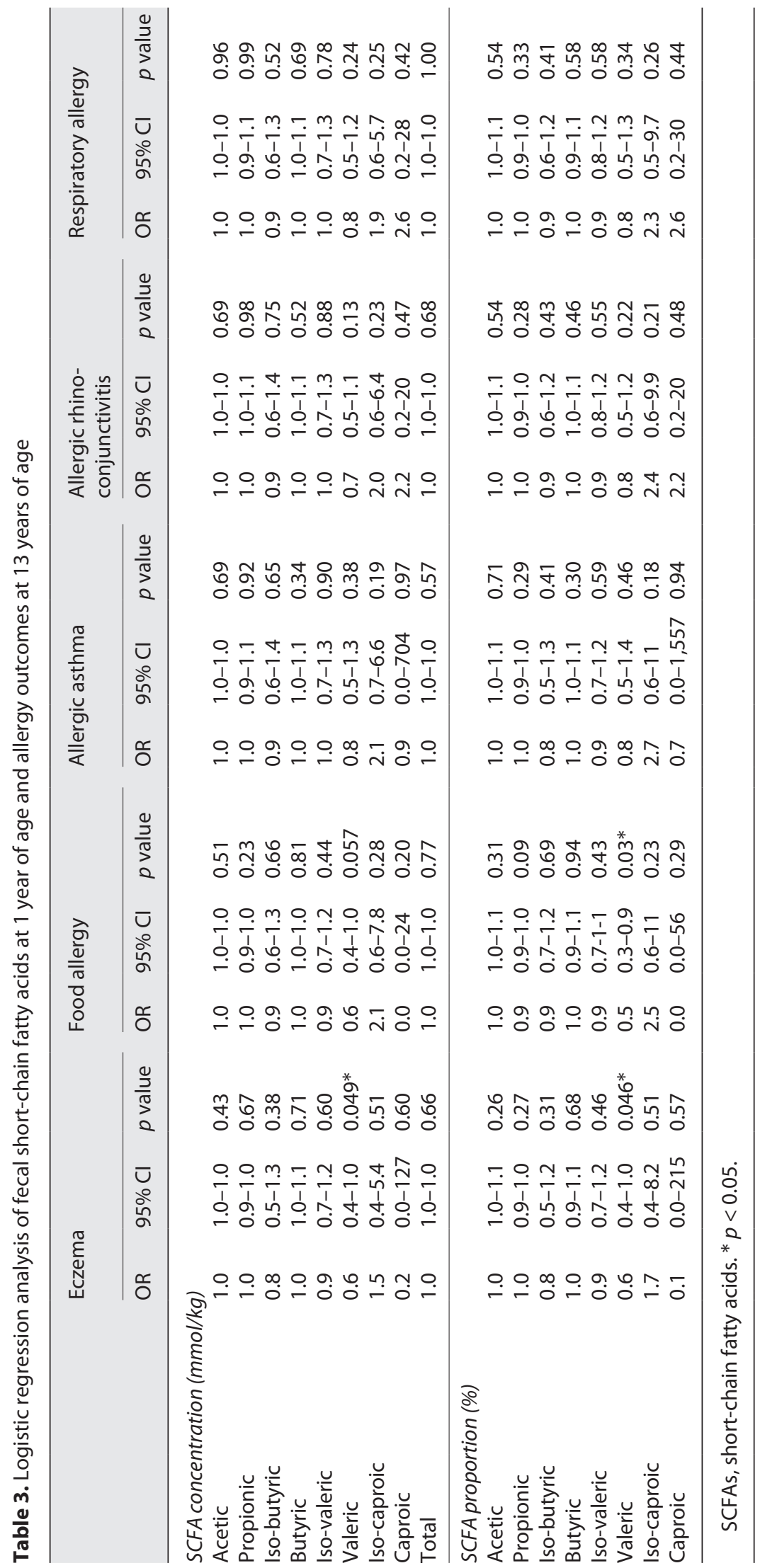


SCFAs produced depend on both the gut bacteria present and the substrate provided to them [38]. However, the concentration of valeric acid at 1 year of age remained non-significantly inversely associated with food allergy at 13 years of age (OR 0.6, 95\% CI: $0.3-1.0, p=0.07$ ) after exclusion of these individuals, and the proportion of valeric acid also remained inversely associated with food allergy (OR 0.5, 95\% CI: 0.3-1.0, $p=0.04$ ).

\section{Fecal SCFAs at 1 Year of Age and Eczema Resolution}

by 13 Years of Age

At 1 year of age, the adolescents in this study were clinically evaluated for allergy by the study pediatrician (AS), as described previously [22], and 14 were diagnosed with eczema. Of these, 7 still had eczema at 13 years of age, while the other 7 saw their eczema resolve. Fecal SCFAs in the group diagnosed with eczema at 1 year of age were related to the presence or absence of eczema at 13 years of age (online suppl. Table 1; for all online suppl. material, see www.karger.com/doi/10.1159/000520149). A higher concentration, or a higher proportion, of valeric acid at 1 year of age was linked with reduced risk of eczema at 13 years of age, although not significantly (OR $0.2,95 \%$ CI: $0.0-1.5, p=0.12$ and OR $0.2,95 \%$ CI: $0.0-1.5$, $p=0.11$, respectively).

Fecal SCFAs at 1 Year of Age and Allergy to Particular Foods at 13 Years of Age

Adolescents with food allergy at 13 years of age reported the particular foods they reacted to. As shown in Table 4 , the most common foods associated with allergic symptoms were tree nuts $(N=10)$, peanuts $(N=6)$, fruits $(N=17)$, milk $(N=5)$, and egg $(N=4)$. The fecal SCFA pattern at 1 year of age was related to allergy to particular foods at 13 years of age, as shown in Table 5 . As previously indicated, individuals who were non-allergic at 13 years of age constituted the reference group. The concentration and proportion of propionic acid were both inversely linked with allergy to tree nuts at age 13 (OR 0.9, 95\% CI: $0.8-1.0, p=0.08$ and OR $0.9,95 \%$ CI: $0.8-1.0$, $p=0.04$, respectively), as were the concentration and proportion of valeric acid (OR $0.3,95 \%$ CI: $0.1-1.1, p=0.06$ and OR $0.2,95 \%$ CI: $0.0-1.0, p=0.04$, respectively). Propionic acid levels at 1 year of age were also inversely related to peanut allergy at 13 years of age (OR $0.9,95 \% \mathrm{CI}$ : $0.7-1.0, p=0.06$ and OR $0.8,95 \%$ CI: $0.6-1.0, p=0.02$ for concentration and proportion, respectively), while butyric acid levels were positively associated with peanut allergy (OR 1.1, 95\% CI: $1.0-1.2, p=0.03$ and OR 1.2, 95\% CI: $1.0-1.3, p=0.03$ for concentration and proportion,
Table 4. Frequency of allergies to particular foods at 13 years of age

\begin{tabular}{llll}
\hline & $\begin{array}{l}\text { All adolescents } \\
\text { with food allergy, } \\
(N=28)\end{array}$ & $\begin{array}{l}\text { Diagnosed year, } \\
(N=9)\end{array}$ & $\begin{array}{l}\text { Diagnosed } \\
(N=13 \text { years, }\end{array}$ \\
& 10 & 4 & 6 \\
Tree nuts & 6 & 3 & 3 \\
Peanuts & & & \\
\hline
\end{tabular}

\begin{tabular}{llll}
\hline Fruits & & & \\
Any fruit & 17 & 4 & 13 \\
Mandarin & 2 & 1 & 1 \\
Orange & 4 & 2 & 2 \\
Apple & 5 & 2 & 3 \\
Kiwi & 12 & 2 & 10 \\
\hline Vegetables & & & \\
Carrot & 1 & 1 & 0 \\
Tomato & 1 & 1 & 0 \\
\hline Other & & & \\
Milk & 5 & 4 & 1 \\
Egg & 4 & 3 & 1 \\
Fish & 1 & 1 & 0 \\
\hline
\end{tabular}

respectively). In addition, the proportion of iso-butyric acid tended to be inversely associated with allergy to milk (OR 0.3, 95\% CI: $0.1-1.2, p=0.08$ ). No analysis was carried out with respect to egg due to the low number of cases.

In a further analysis, adolescents with food allergies diagnosed by 1 year of age were excluded to avoid any effect of food elimination diets on the fecal SCFA pattern at 1 year of age. Among adolescents with food allergy diagnosed after 1 year of age, 6 were allergic to tree nuts, 3 to peanuts, 13 to fruits, 1 to milk, and 1 to egg (shown in Table 4). No significant associations between fecal SCFA pattern at 1 year of age and allergy to particular foods at 13 years of age were observed, as shown in online supplementary Table 2. However, the concentration and proportion of iso-caproic acid were non-significantly associated with allergy to tree nuts (OR 4.7, 95\% CI: $0.8-28, p=$ 0.09 and OR 6.4, 95\% CI: $1.0-41, p=0.050$, respectively).

Factors Associated with Eczema and Food Allergy at 13 Years of Age, and with Fecal Valeric Acid at 1 Year of Age

Factors associated with eczema and food allergy at 13 years of age were examined using data collected by questionnaire on early life exposures and parental allergy, as shown in online supplementary Table 3. Maternal allergy, defined as eczema, asthma, or hay fever, was associated with eczema (OR 6.4, 95\% CI: 1.7-23, $p=0.005$ ), and also 
Table 5. Logistic regression analysis of fecal SCFAs at 1 year of age and allergy to particular foods at 13 years of age

\begin{tabular}{|c|c|c|c|c|c|c|c|c|c|c|c|c|}
\hline & OR & $95 \% \mathrm{Cl}$ & $p$ value & OR & $95 \% \mathrm{Cl}$ & $p$ value & OR & $95 \% \mathrm{Cl}$ & $p$ value & OR & $95 \% \mathrm{Cl}$ & $p$ value \\
\hline Acetic & 1.0 & $1.0-1.0$ & 0.35 & 1.0 & $1.0-1.1$ & 0.15 & 1.0 & $1.0-1.0$ & 0.93 & 1.0 & $1.0-1.0$ & 0.52 \\
\hline Propionic & 0.9 & $0.8-1.0$ & 0.08 & 0.9 & $0.7-1.0$ & 0.06 & 1.0 & $0.9-1.0$ & 0.26 & 1.0 & $0.9-1.1$ & 0.85 \\
\hline Iso-butyric & 0.7 & $0.4-1.4$ & 0.34 & 0.5 & $0.2-1.4$ & 0.19 & 1.1 & $0.7-1.6$ & 0.67 & 0.4 & $0.1-1.4$ & 0.15 \\
\hline Valeric & 0.3 & $0.1-1.1$ & 0.06 & 0.4 & $0.1-1.5$ & 0.16 & 0.7 & $0.4-1.2$ & 0.18 & 0.7 & $0.3-1.6$ & 0.37 \\
\hline Iso-caproic & 2.9 & $0.7-13$ & 0.15 & 2.6 & $0.7-10$ & 0.15 & 1.6 & $0.3-9.4$ & 0.63 & - & - & - \\
\hline Total & 1.0 & $1.0-1.0$ & 0.69 & 1.0 & $1.0-1.0$ & 0.22 & 1.0 & $1.0-1.0$ & 0.95 & 1.0 & $1.0-1.0$ & 0.70 \\
\hline \multicolumn{13}{|c|}{ SCFA proportion (\%) } \\
\hline Acetic & 1.1 & $1.0-1.1$ & 0.14 & 1.1 & $1.0-1.2$ & 0.28 & 1.0 & $1.0-1.1$ & 0.71 & 1.0 & $0.9-1.2$ & 0.37 \\
\hline Valeric & 0.2 & $0.0-1.0$ & $0.04^{*}$ & 0.2 & $0.0-1.3$ & 0.10 & 0.6 & $0.4-1.2$ & 0.14 & 0.5 & $0.2-1.5$ & 0.21 \\
\hline Iso-caproic & 3.8 & $0.7-20$ & 0.11 & 3.2 & $0.6-16$ & 0.16 & 1.8 & $0.3-11$ & 0.52 & - & - & - \\
\hline
\end{tabular}

A dash (-) indicates the SCFA was not detected in any individuals with allergy to the particular food. Caproic acid not included, as detected in $\leq 1$ individual with each allergy. SCFAs, short-chain fatty acids. ${ }^{*} p<0.05$.

with food allergy (OR 3.5, 95\% CI: 1.0-12, $p=0.04$ ). The associations between paternal allergy and either eczema or food allergy were weaker and not significant (OR 1.8, 95\% CI: $0.5-6.5, p=0.35$ and OR 1.5, 95\% CI: 0.5-4.9, $p$ $=0.47$ ). Girls had a non-significantly lower likelihood than boys of eczema and food allergy, as compared to being non-allergic (OR 0.5, 95\% CI: $0.2-1.4, p=0.17$ and OR $0.4,95 \%$ CI: $0.2-1.2, p=0.10$, respectively).

Factors associated with the fecal valeric acid concentration at 1 year of age were also examined, using the same data as above, as shown in online supplementary Table 4 . Parental allergy tended to be associated with lower fecal valeric acid concentration at 1 year of age (B - $0.5,95 \%$ CI: -0.9 to $0.0, p=0.07$ ), with a similar relationship for both maternal and paternal allergy. Being a girl showed a weak and non-significant positive association with valeric acid concentrations $(p=0.21)$. Factors such as exclusive breastfeeding at 6 months of age, antibiotic treatment or symptoms of at least 3 infectious diseases during the first year, birth weight, elder siblings, or ownership of a furred pet were not significantly related to valeric acid concentration.

To further examine relationships between host factors, fecal SCFA pattern at 1 year of age, and allergy at 13 years, we performed separate logistic regression models of fecal
SCFAs in relation to eczema and food allergy, stratified by various host factors. When stratified by parental allergy, valeric acid concentration was inversely associated with eczema and food allergy in the group with allergic parents $(N=70$; OR $0.5,95 \%$ CI: $0.3-1.0, p=0.047$ and OR 0.4, $95 \%$ CI: $0.2-0.9, p=0.03$, respectively, online suppl. Table 5). Stratified by sex, valeric acid concentration was inversely associated with eczema among girls $(N=46$; OR $0.4,95 \%$ CI: $0.2-0.9, p=0.03$, online suppl. Table 6) and a similar tendency was seen for food allergy (OR 0.6, 95\% CI: $0.3-1.1, p=0.09)$. Along the same lines, stratification for elder siblings revealed that propionic and valeric acid concentration tended to be negatively associated with food allergy in children with no elder siblings $(N=40$; OR $0.8,95 \%$ CI: $0.7-1.0, p=0.06$ and OR $0.4,95 \%$ CI: $0.1-1.1$, $p=0.08$, respectively, online suppl. Table 7 ), and valeric acid concentration also appeared to be negatively associated with eczema (OR 0.5, 95\% CI: 0.2-1.2, $p=0.10$ ). Stratification for exclusive breastfeeding at 6 months similarly revealed inverse relationships between propionic and valeric acid concentration and food allergy in those individuals who received formula and/or other foods beside breast milk ( $N=52$; OR 0.9, 95\% CI: $0.8-1.0, p=0.008$ and OR 0.5, 95\% CI: $0.3-1.0, p=0.06$, respectively, online suppl. Table 8 ), as well as a possible negative association 
between valeric acid concentration and eczema (OR 0.5, 95\% CI: $0.2-1.1, p=0.10)$. In contrast, no significant associations were observed between fecal SCFA pattern at 1 year of age and either eczema and food allergy at 13 years among individuals with non-allergic parents (although this group included relatively few cases) or elder siblings, boys, or those who were exclusively breastfed at 6 months.

\section{Discussion}

A key finding of our study was that the concentration of valeric acid in feces at 1 year of age, or proportion of this particular SCFA among the total level of SCFA in feces, was inversely related to questionnaire-reported eczema and food allergy at 13 years of age. We also observed that among infants with eczema at 1 year of age, those who had a higher fecal valeric acid concentration appeared to have a lower risk of their condition persisting in adolescence. However, this finding included relatively few individuals and did not reach statistical significance. Both eczema and food allergy often present during early childhood, although they may be outgrown and replaced with other allergic conditions, such as asthma and allergic rhinitis, a phenomenon termed "the atopic march." However, eczema continues into adulthood in around $40 \%$ of cases [39]. Epidemiological evidence on valeric acid in relation to allergy remains limited, as studies in the field have often not included this SCFA in their analysis. However, our findings are consistent with data from the Swedish FARMFLORA birth cohort, which showed that school-age children with eczema had lower median levels of valeric acid in their feces at 3 years of age than non-allergic children [18]. Correspondingly, a recent Korean study also observed lower levels of fecal valeric acid, as well as butyric acid, in infants with eczema at 6 months of age [27].

Food allergy is notoriously difficult to diagnose and reliable diagnosis requires elimination-provocation, preferably carried out with blinding of the patient and observer. In our study, diagnosis was based on questionnaire-reported food allergy and is therefore somewhat tentative. Many food allergies, particularly to milk and eggs, usually wane, while allergy to tree nuts and fish usually persist into adulthood. Consistent with this, allergies to tree nuts and peanuts were common among the adolescents in this cohort, as also were fruit allergies. We observed inverse relationships between the concentration and/or proportion of fecal propionic and valeric acid at 1 year of age and reported tree nut allergy at 13 years of age, and also associations between propionic and butyric acid

SCFAs in Infancy and Allergy in

Adolescence at 1 year of age and reported peanut allergy at 13 years of age. These associations did not remain after exclusion of adolescents who had already been diagnosed with food allergy at 1 year of age, to avoid the possibility of food elimination diets affecting fecal SCFA patterns [38], although only a relatively small number of cases were included. However, a tendency for a higher concentration and proportion of iso-caproic acid at 1 year of age to be associated with allergy to tree nuts at 13 years was observed in this latter analysis. Iso-caproic acid is a metabolite of $C$. difficile [21], a bacterium that thrives in poorly developed microbiota, for instance, during infancy [9] and in adults after treatment with broad-spectrum antibiotics [40], and has been linked with atopy [35].

Previously in this birth cohort, we observed that 1 year-old infants diagnosed with allergy had lower concentrations of propionic, iso-butyric, butyric, iso-valeric, and valeric acid in their feces than non-allergic infants, and higher concentrations of iso-caproic acid. Low concentrations of fecal iso-butyric, valeric, and iso-valeric acid at 1 year of age were also associated with onset of food allergy between 1 and 4 years of age [22]. In contrast, only valeric acid was associated with any of the principal allergy diagnoses included in this study at 13 years of age. An altered diet may explain at least some of the SCFA pattern observed among 1-year-old allergic children. Further, there were various methodological differences between the studies in this series, including differences in sample size, allergic manifestation(s) investigated, and diagnosis method. However, our observations of SCFA patterns having altered links with allergy at different time points may also relate to the changing profile of allergy over time. Many allergic children "grow out" of their allergy during their early years, and it may be the case that different SCFA patterns are present in children who either do or do not acquire tolerance.

Taken together, our findings point to valeric acid being of particular interest as a marker of a microbiota that may protect against allergy. In the current study, valeric acid concentration could not be attributed to any of the host factors for which data were collected, although having an allergic parent was associated with non-significantly lower levels of valeric acids in feces. Previously, this SCFA has been associated with elder siblings, including in an earlier study based on the BAS cohort $[18,22]$. While no such association was observed here, this study comprised a sub-set of children included in the previous BAS study and the median valeric acid concentration remained somewhat higher among children with elder siblings (0.66 vs. $0.22 \mathrm{mmol} / \mathrm{kg}$ ). Factors such as wearing 
shoes indoors, higher indoor humidity, and living in an older house have been associated with reduced relative abundance of Streptococcaceae in the home microbiota [27], while the proportion of fecal Streptococcus has been negatively correlated with valeric and also butyric acid levels [41]. It may be that such exposures contributed to valeric acid concentrations in our study, although no data were collected on these parameters. To further investigate the relationship between fecal SCFAs, allergy, and host factors included in our study, stratified analyses were carried out. The relationship between fecal SCFA pattern and eczema or food allergy observed in the whole cohort was most apparent among individuals with allergic parents, and seemingly also in children with no elder siblings, those who were not exclusively breastfed at 6 months of age, and in girls. An inverse relationship between fecal propionic acid at 1 year and food allergy at 13 years also emerged in certain sub-groups. Therefore, the inverse relationship between an SCFA pattern characterized by higher levels of fecal valeric acid at 1 year of age and eczema and food allergy at 13 years of age was modified by host parameters, and may be especially evident in children who lack other protective factors.

Valeric acid is generated via the bacterial breakdown of amino acids such as proline and hydroxyproline [42]. It also derives from lactic and propionic acid, which are taken up by secondary metabolizers and used as substrates in chain-lengthening reactions to produce valeric acid [43, 44]. Therefore, higher levels of fecal valeric acid at 1 year of age may reflect a more mature gut microbiota containing a greater amount of such cross-feeding activity. Furthermore, certain species and/or strains of bacteria from the genera Clostridium [45], Eubacterium [46], Oscillibacter [47], and Megasphaera [48] produce valeric acid, suggesting a greater abundance of such bacteria in the gut microbiota of non-allergic participants in our study at 1 year of age. However, studies relating gut microbiota composition to allergy have generated somewhat inconsistent results and the specific bacteria relevant to allergy development have yet to be fully resolved [49].

An intriguing possibility is that valeric acid may be an active agent in helping reduce the risk of allergy. Until recently, little was known about the physiological properties of valeric acid. Research focused primarily on acetic, propionic, and butyric acids, as these are the most abundant SCFAs in the human gut [20]. However, valeric acid may be an immune regulator, inducing production of IL10 and suppressing release of the pro-inflammatory cytokine IL-17A in CD4+ T-cells, and also inducing production of IL-10 in regulatory B cells [50]. These effects occur through the ability of valeric acid to reprogram lymphocyte metabolism toward increased glucose oxidation, and also through its activity as a potent histone deacetylase inhibitor [50,51]. Despite this evidence, it might be questioned whether the concentration of valeric acid in the gut is sufficient to produce a biological effect, as it is found at substantially lower levels than the major SCFAs. However, its histone deacetylase activity was demonstrated in vitro at levels of just $5 \mathrm{mM}$, similar to the highest concentrations of valeric acid (up to $5.6 \mathrm{mmol} / \mathrm{kg}$ of feces) we observed in this study. Therefore, it is possible that valeric acid may play an active role in suppressing inflammatory responses and establishing immune tolerance.

This study adds to current knowledge on fecal SCFA patterns in relation to allergy, and is the first to our knowledge that has described fecal SCFA patterns during early childhood with respect to allergy outcomes in adolescence. A limitation of our study is that allergy was established using participants' responses to a questionnaire, rather that direct diagnosis by a physician. It should also be noted that, although the BAS birth cohort aimed to include all the children born at Östersund hospital in northern Sweden during a period of 1 year and was therefore broadly representative of the local population, the present study was based on a subgroup previously selected for SCFA analysis. In selecting this sub-group, children with sensitization at 1 year of age were favored, leading to a higher rate of allergy in adolescence than seen in the cohort as a whole. While this facilitated the analysis of SCFA levels in relation to allergy outcomes, it may also limit the generalizability of our findings.

In conclusion, higher levels of valeric acid in feces during infancy, reflecting an altered metabolic activity of the gut microbiota, were inversely associated with questionnaire-reported eczema and food allergy in adolescence. Higher levels of fecal valeric acid may be characteristic of a protective gut microbiota and/or actively contribute to allergy protection. Our findings lend further support to the notion of early childhood as a critical period when allergy may be programmed via the gut microbiota.

\section{Acknowledgments}

We thank Anna Bernholm at Östersund Hospital for collecting the fecal samples and performing the skin prick tests, Anna-Karin Persson at the Karolinska Institute for performing the gas chromatography analyses, and Robert Lundqvist at Sunderbyn Hospital for statistical support. We also thank all children and parents for participating in this study. 


\section{Statement of Ethics}

This study was conducted according to the standards laid out in the Declaration of Helsinki. Ethical approval was granted by the Regional Ethical Committee for Human Research at the University Hospital, Umeå, Sweden (No. 95-149, 2009-1116-31 and 2012163-32M) and written consent was obtained from parents. At 13 years of age, verbal approval for the skin prick test was also obtained from the adolescents, and the adolescents answered the questionnaire jointly with the parents.

\section{Conflict of Interest Statement}

A.E.W. holds shares in Flora Innovation, a small researcherdriven company investigating potential preventive treatments against immune-regulated diseases. The other authors have no conflicts of interest to declare.

\section{Funding Sources}

This study was supported by grants from the Swedish Foundation for Health Care Science, the Swedish Environmental Protection Agency, the Research and Development unit of the County
Council in Jämtland, the County Councils in Northern Sweden, Queen Silvia's Jubilee Foundation, and Västra Götaland Region (ALFGBG1384019). The funding sources had no role in the preparation of data or the manuscript.

\section{Author Contributions}

A.S. conceived and supervised the BAS birth cohort. A.S. and L.B. designed this study, which M.B. and A.E.W. jointly supervised. K.S. and M.G-B. analyzed the data, and M.G-B. wrote the manuscript. A.S., M.B., A.E.W., L.B., E.N., and B.B. revised the work for important intellectual content. All authors reviewed the manuscript.

\section{Data Availability Statement}

The datasets generated during and/or analyzed during the current study are available from the corresponding author on reasonable request.

\section{References}

1 Jackson KD, Howie LD, Akinbami LJ. Trends in allergic conditions among children: United States, 1997-2011. NCHS Data Brief. 2013; (121):1-8.

2 Pawankar R. Allergic diseases and asthma: a global public health concern and a call to action. World Allergy Organ J. 2014;7(1):12.

3 Bråbäck L, Vogt H, Hjern A. Migration and asthma medication in international adoptees and immigrant families in Sweden. Clin Exp Allergy. 2011;41(8):1108-15.

4 Hjern A, Rasmussen F, Hedlin G. Age at adoption, ethnicity and atopic disorder: a study of internationally adopted young men in Sweden. Pediatr Allergy Immunol. 1999; 10(2):101-6.

5 Matricardi PM, Rosmini F, Riondino S, Fortini M, Ferrigno L, Rapicetta M, et al. Exposure to foodborne and orofecal microbes versus airborne viruses in relation to atopy and allergic asthma: epidemiological study. BMJ. 2000;320(7232):412-7.

6 Matricardi PM, Rosmini F, Panetta V, Ferrigno L, Bonini S. Hay fever and asthma in relation to markers of infection in the United States. J Allergy Clin Immunol. 2002;110(3): 381-7.

7 Matricardi PM, Rosmini F, Ferrigno L, Nisini $\mathrm{R}$, Rapicetta M, Chionne P, et al. Cross sectional retrospective study of prevalence of atopy among Italian military students with antibodies against hepatitis A virus. BMJ. 1997; 314(7086):999-1003.
8 Adlerberth I, Jalil F, Carlsson B, Mellander L, Hanson LA, Larsson P, et al. High turnover rate of Escherichia coli strains in the intestinal flora of infants in Pakistan. Epidemiol Infect. 1998;121(3):587-98.

9 Adlerberth I, Wold AE. Establishment of the gut microbiota in Western infants. Acta Paediatr. 2009;98(2):229-38.

10 Adlerberth I, Lindberg E, Aberg N, Hesselmar $\mathrm{B}$, Saalman R, Strannegård IL, et al. Reduced enterobacterial and increased staphylococcal colonization of the infantile bowel: an effect of hygienic lifestyle? Pediatr Res. 2006;59(1): 96-101.

11 Wang M, Karlsson C, Olsson C, Adlerberth I, Wold AE, Strachan DP, et al. Reduced diversity in the early fecal microbiota of infants with atopic eczema. J Allergy Clin Immunol. 2008;121(1):129-34.

12 Bisgaard H, Li N, Bonnelykke K, Chawes BL, Skov T, Paludan-Müller G, et al. Reduced diversity of the intestinal microbiota during infancy is associated with increased risk of allergic disease at school age. J Allergy Clin Immunol. 2011;128(3):646-52.e1-5.

13 Ismail IH, Oppedisano F, Joseph SJ, Boyle RJ, Licciardi PV, Robins-Browne RM, et al. Reduced gut microbial diversity in early life is associated with later development of eczema but not atopy in high-risk infants. Pediatr Allergy Immunol. 2012;23(7):674-81.

14 Björkstén B, Sepp E, Julge K, Voor T, Mikelsaar M. Allergy development and the intesti- nal microflora during the first year of life. J Allergy Clin Immunol. 2001;108(4):516-20.

15 Corrêa-Oliveira R, Fachi JL, Vieira A, Sato FT, Vinolo MA. Regulation of immune cell function by short-chain fatty acids. Clin Transl Immunology. 2016;5(4):e73.

16 Tan J, McKenzie C, Potamitis M, Thorburn AN, Mackay CR, Macia L. The role of shortchain fatty acids in health and disease. Adv Immunol. 2014;121:91-119.

17 Midtvedt AC, Midtvedt T. Production of short chain fatty acids by the intestinal microflora during the first 2 years of human life. J Pediatr Gastroenterol Nutr. 1992;15(4):395-403.

18 Gio-Batta M, Sjöberg F, Jonsson K, Barman M, Lundell AC, Adlerberth I, et al. Fecal short chain fatty acids in children living on farms and a link between valeric acid and protection from eczema. Sci Rep. 2020;10(1):22449.

19 Bäckhed F, Roswall J, Peng Y, Feng Q, Jia H, Kovatcheva-Datchary $\mathrm{P}$, et al. Dynamics and stabilization of the human gut microbiome during the first year of life. Cell Host Microbe. 2015;17(6):852.

20 Cummings JH, Pomare EW, Branch WJ, Naylor CP, Macfarlane GT. Short chain fatty acids in human large intestine, portal, hepatic and venous blood. Gut. 1987;28(10):1221-7.

21 Böttcher MF, Nordin EK, Sandin A, Midtvedt T, Björkstén B. Microflora-associated characteristics in faeces from allergic and nonallergic infants. Clin Exp Allergy. 2000;30(11): 1590-6. 
22 Sandin A, Bråbäck L, Norin E, Björkstén B. Faecal short chain fatty acid pattern and allergy in early childhood. Acta Paediatr. 2009; 98(5):823-7.

23 Depner M, Taft DH, Kirjavainen PV, Kalanetra KM, Karvonen AM, Peschel S, et al. Maturation of the gut microbiome during the first year of life contributes to the protective farm effect on childhood asthma. Nat Med. 2020; 26(11):1766-75.

24 Wopereis H, Sim K, Shaw A, Warner JO, Knol J, Kroll JS. Intestinal microbiota in infants at high risk for allergy: effects of prebiotics and role in eczema development. J Allergy Clin Immunol. 2018;141(4):1334-42.e5.

25 Ta LDH, Chan JCY, Yap GC, Purbojati RW, Drautz-Moses DI, Koh YM, et al. A compromised developmental trajectory of the infant gut microbiome and metabolome in atopic eczema. Gut microbes. 2020;12(1):1-22.

26 Song H, Yoo Y, Hwang J, Na YC, Kim HS. Faecalibacterium prausnitzii subspecies-level dysbiosis in the human gut microbiome underlying atopic dermatitis. J Allergy Clin Immunol. 2016;137(3):852-60.

27 Kang M-J, Lee S-Y, Park Y-M, Kim B-S, Lee $\mathrm{M}-\mathrm{J}, \mathrm{Kim} \mathrm{J}-\mathrm{H}$, et al. Interactions between IL17 variants and streptococcus in the gut contribute to the development of atopic dermatitis in infancy. Allergy Asthma Immunol Res. 2021;13(3):404-19.

28 Kim HK, Rutten NB, Besseling-van der Vaart I, Niers LE, Choi YH, Rijkers GT, et al. Probiotic supplementation influences faecal short chain fatty acids in infants at high risk for eczema. Benef Microbes. 2015;6(6):783-90.

29 Thompson-Chagoyan OC, Fallani M, Maldonado J, Vieites JM, Khanna S, Edwards C, et al. Faecal microbiota and short-chain fatty acid levels in faeces from infants with cow's milk protein allergy. Int Arch Allergy Immunol. 2011;156(3):325-32.

30 Diaz M, Guadamuro L, Espinosa-Martos I, Mancabelli L, Jimenez S, Molinos-Norniella $\mathrm{C}$, et al. Microbiota and derived parameters in fecal samples of infants with non-IgE cow's milk protein allergy under a restricted diet. Nutrients. 2018;10(10):1481.
31 Berni Canani R, De Filippis F, Nocerino R, Paparo L, Di Scala C, Cosenza L, et al. Gut microbiota composition and butyrate production in children affected by non-IgE-mediated cow's milk allergy. Sci Rep. 2018;8(1): 12500.

32 Goldberg MR, Mor H, Magid Neriya D, Magzal F, Muller E, Appel MY, et al. Microbial signature in IgE-mediated food allergies. Genome Med. 2020;12(1):92.

33 Chiu CY, Cheng ML, Chiang MH, Kuo YL, Tsai MH, Chiu CC, et al. Gut microbial-derived butyrate is inversely associated with IgE responses to allergens in childhood asthma. Pediatr Allergy Immunol. 2019;30(7):689-97.

34 Roduit C, Frei R, Ferstl R, Loeliger S, Westermann P, Rhyner C, et al. High levels of butyrate and propionate in early life are associated with protection against atopy. Allergy. 2019; 74(4):799-809.

35 Vu K, Lou W, Tun HM, Konya TB, MoralesLizcano N, Chari RS, et al. From birth to overweight and atopic disease: multiple and common pathways of the infant gut microbiome. Gastroenterology. 2021;160(1):128-44.e10.

36 Sandin A, Björkstén B, Bråbäck L. Development of atopy and wheezing symptoms in relation to heredity and early pet keeping in a Swedish birth cohort. Pediatr Allergy Immunol. 2004;15(4):316-22.

37 Asher MI, Keil U, Anderson HR, Beasley R, Crane J, Martinez F, et al. International study of asthma and allergies in childhood (ISAAC): rationale and methods. Eur Respir J. 1995; 8(3):483-91.

38 Zarling EJ, Ruchim MA. Protein origin of the volatile fatty acids isobutyrate and isovalerate in human stool. J Lab Clin Med. 1987;109(5): 566-70.

39 Bantz SK, Zhu Z, Zheng T. The atopic march: progression from atopic dermatitis to allergic rhinitis and asthma. J Clin Cell Immunol. 2014;5(2):202.

40 Mullish BH, Williams HR. Clostridium difficile infection and antibiotic-associated diarrhoea. Clin Med. 2018;18(3):237-41.

41 Kirjavainen PV, Karvonen AM, Adams RI, Täubel M, Roponen $\mathrm{M}$, Tuoresmäki $\mathrm{P}$, et al. Farm-like indoor microbiota in non-farm homes protects children from asthma development. Nat Med. 2019;25(7):1089-95.
42 Rasmussen HS, Holtug K, Mortensen PB. Degradation of amino acids to short-chain fatty acids in humans. An in vitro study. Scand J Gastroenterol. 1988;23(2):178-82.

43 Jeon BS, Choi O, Um Y, Sang BI. Production of medium-chain carboxylic acids by Megasphaera sp. MH with supplemental electron acceptors. Biotechnol Biofuels. 2016;9:129.

44 Bourriaud C, Robins RJ, Martin L, Kozlowski $\mathrm{F}$, Tenailleau E, Cherbut C, et al. Lactate is mainly fermented to butyrate by human intestinal microfloras but inter-individual variation is evident. J Appl Microbiol. 2005;99(1): 201-12.

45 Elsden SR, Hilton MG. Volatile acid production from threonine, valine, leucine and isoleucine by clostridia. Arch Microbiol. 1978; 117(2):165-72.

46 Wallace RJ, McKain N, McEwan NR, Miyagawa E, Chaudhary LC, King TP, et al. Eubacterium pyruvativorans sp. nov., a novel nonsaccharolytic anaerobe from the rumen that ferments pyruvate and amino acids, forms caproate and utilizes acetate and propionate. Int J Syst Evol Microbiol. 2003;53(Pt 4):96570.

47 Iino T, Mori K, Tanaka K, Suzuki KI, Harayama S. Oscillibacter valericigenes gen. nov., sp. nov., a valerate-producing anaerobic bacterium isolated from the alimentary canal of a Japanese corbicula clam. Int J Syst Evol Microbiol. 2007;57(Pt 8):1840-5.

48 Lanjekar VB, Marathe NP, Ramana VV, Shouche YS, Ranade DR. Megasphaera indica sp. nov., an obligate anaerobic bacteria isolated from human faeces. Int J Syst Evol Microbiol. 2014;64(Pt 7):2250-6.

49 Petersen EBM, Skov L, Thyssen JP, Jensen P. Role of the gut microbiota in atopic dermatitis: a systematic review. Acta Derm Venereol. 2019;99(1):5-11.

50 Luu M, Pautz S, Kohl V, Singh R, Romero R, Lucas $S$, et al. The short-chain fatty acid pentanoate suppresses autoimmunity by modulating the metabolic-epigenetic crosstalk in lymphocytes. Nat Commun. 2019;10(1):760.

51 Yuille S, Reichardt N, Panda S, Dunbar H, Mulder IE. Human gut bacteria as potent class I histone deacetylase inhibitors in vitro through production of butyric acid and valeric acid. PLoS One. 2018;13(7):e0201073. 